\title{
Possible discrepancy of HbA1c values and its assessment among patients with chronic renal failure, hemodialysis and other diseases
}

\author{
Kaori Inoue $^{1,3}$ - Atsushi Goto ${ }^{2,3}$ - Miyako Kishimoto ${ }^{1}$. Tetsuro Tsujimoto ${ }^{1}$. \\ Ritsuko Yamamoto-Honda ${ }^{1} \cdot$ Hiroshi Noto $^{1} \cdot$ Hiroshi Kajio $^{1} \cdot$ Yasuo Terauchi $^{3}$. \\ Mitsuhiko Noda ${ }^{2}$
}

Received: 9 October 2014/ Accepted: 22 March 2015/Published online: 1 April 2015

(c) The Author(s) 2015. This article is published with open access at Springerlink.com

\begin{abstract}
Background Glycated hemoglobin (HbA1c) and glycated albumin (GA) are frequently used as glycemic control markers. However, these markers are influenced by alterations in hemoglobin and albumin metabolism. Thus, conditions such as anemia, chronic renal failure, hypersplenism, chronic liver diseases, hyperthyroidism, hypoalbuminemia, and pregnancy need to be considered when interpreting $\mathrm{HbA} 1 \mathrm{c}$ or GA values. Using data from patients with normal albumin and hemoglobin metabolism, we previously established a linear regression equation describing the GA value versus the $\mathrm{HbA} 1 \mathrm{c}$ value to calculate an extrapolated $\mathrm{HbAlc}(\mathrm{eHbA1c})$ value for the accurate evaluation of glycemic control. In this study, we investigated the difference between the measured $\mathrm{HbAlc}$ and the eHbA1c values for patients with various conditions.

Methods Data sets for a total of 2461 occasions were obtained from 731 patients whose HbA1c and GA values were simultaneously measured. We excluded patients with missing data or changeable $\mathrm{HbA1c}$ levels, and patients who had received transfusions or steroids within the previous 3 months. Finally, we included 44 patients with chronic renal failure (CRF), 10 patients who were undergoing
\end{abstract}

Mitsuhiko Noda

mnoda@hosp.ncgm.go.jp

1 Department of Endocrinology, Diabetes, and Metabolism, Center Hospital, National Center for Global Health and Medicine, Tokyo 162-8655, Japan

2 Department of Diabetes Research, National Center for Global Health and Medicine, 1-21-1 Toyama, Shinjuku-ku, Tokyo 162-8655, Japan

3 Department of Endocrinology and Metabolism, Yokohama City University Graduate School of Medicine, Yokohama 236-0004, Japan hemodialysis (HD), 7 patients with hematological malignancies and a hemoglobin level of less than $10 \mathrm{~g} / \mathrm{dL}$ (HM), and 12 patients with chronic liver diseases (CLD).

Results In all the groups, the eHbA1c values were significantly higher than the measured $\mathrm{HbAlc}$ values. The median difference was $0.75 \%$ (95\% CI $0.40-1.10 \%$, $P$ for the difference is $<0.001)$ in the CRF group, $0.80 \%$ (95\% CI $0.30-1.65 \%, P$ for the difference is 0.041 ) in the HD group, $0.90 \%(95 \%$ CI $0.90-1.30 \%, P$ for the difference is 0.028$)$ in the HM group, and $0.85 \%(95 \% \mathrm{CI}$ $0.40-1.50 \%, P$ for the difference is 0.009 ) in the CLD group.

Conclusions We found that the measured $\mathrm{HbA} 1 \mathrm{c}$ values were lower than the eHbAlc values in each of the groups.

Keywords Glycated hemoglobin - Glycated albumin . Chronic renal failure

\section{Introduction}

Glycated hemoglobin (HbA1c) and glycated albumin (GA) are frequently used as glycemic control markers. HbAlc is used as the gold standard index of glycemic control in clinical practice for diabetes treatment [1]. Since the lifespan of erythrocytes is approximately 120 days, HbA1c reflects the plasma glucose levels over the past few months. The metabolic turnover of albumin is faster than hemoglobin, with a lifespan of approximately 17-23 days. Accordingly, GA is used as an index of short-term glycemic control [2].

Although these glycemic control markers are well correlated with blood glucose levels, HbA1c is influenced by alterations in hemoglobin metabolism and GA is influenced by alterations in albumin metabolism. In clinical practice, 
conditions such as anemia, chronic renal failure, hypersplenism, chronic liver diseases, hyperthyroidism, hypoalbuminemia, and pregnancy need to be considered when interpreting $\mathrm{HbA} 1 \mathrm{c}$ or GA values.

In a previous study, we developed a linear regression equation describing the GA value versus the HbA1c value among participants without altered albumin metabolism or hemoglobin metabolism, to calculate an extrapolated HbAlc (eHbA1c) value for the accurate evaluation of glycemic control [3].

We often encounter patients with conditions affecting the turnover of either $\mathrm{HbA} 1 \mathrm{c}$ or GA. In such patients, the measured $\mathrm{HbA} 1 \mathrm{c}$ and GA values are likely to diverge from the equation. Earlier studies have evaluated the associations between mean blood glucose levels, HbA1c values, and GA values in patients on dialysis or patients with chronic liver diseases or hemolytic anemia [4-6]. However, the impact of each condition affecting the turnover of either $\mathrm{HbA} 1 \mathrm{c}$ or GA on the direction and magnitude of the discrepancy between the measured HbAlc and eHbAlc, which is the equation developed in patients who were free of such conditions is not well understood. In this study, we investigated the differences between the measured $\mathrm{HbA} 1 \mathrm{c}$ and the eHbA1c values in patients with various conditions.

\section{Materials and methods}

A flow diagram depicting this study is shown in Fig. 1. We retrospectively analyzed the medical charts of patients attending the National Center for Global Health and Medicine (Tokyo, Japan) during 2011, and selected data sets for a total of 2461 occasions from 731 patients (including nondiabetes patients) whose HbA1c and GA values were simultaneously measured. If these values were measured in a single patient on more than one occasion, we selected the data set containing the smallest $\mathrm{HbAlc}$ value.

We excluded patients whose previous $\mathrm{HbAlc}$ values were missing or whose $\mathrm{HbA} 1 \mathrm{c}$ levels were changeable and selected 550 patients. We then excluded patients without albumin, hemoglobin or eGFR data, and patients who had been treated with transfusions or steroids within the previous 3 months. Finally, we included 44 predialysis patients with an eGFR of less than $30 \mathrm{~mL} / \mathrm{min} / 1.73 \mathrm{~m}^{2}$ chronic renal failure (CRF), 10 patients who were undergoing hemodialysis (HD), 7 patients with hematological malignancies and their hemoglobin level of less than $10 \mathrm{~g} /$ $\mathrm{dL}$ (HM), and 12 patients with chronic liver diseases (CLD). We further excluded patients who had combinations of these diseases, since the aim of this study was to investigate the impact of each condition on the turnover of either $\mathrm{HbA} 1 \mathrm{c}$ or GA, as well as the direction and magnitude of the discrepancy. We did not include patients who were pregnant or who had hyperthyroidism because the data was insufficient for an analysis.

$\mathrm{HbA1c}$ was measured using high-performance liquid chromatography (HPLC) (ARKRAY ADAMS-A1C HA8160; Kyoto, Japan) and was corrected to the National Glycohemoglobin Standardization Program (NGSP) values [7]. GA was measured using an enzymatic method with albumin-specific proteinase, ketoamine oxidase, and an albumin assay reagent (Lucica GA-L; Asahi Kasei Pharma Co., Tokyo, Japan) using an autoanalyzer (Hitachi 770; Hitachi Instruments Service Co., Tokyo, Japan). Each patient was assessed for clinical features such as age, sex, height, body weight, body mass index, blood and urine sample data, history and duration of diabetes mellitus, medications, and complications based on the data contained in the medical records.

This study was approved by the institutional ethical committee of the National Center for Global Health and Medicine (approval number: 1141) and was performed in accordance with the Declaration of Helsinki.

\section{Statistical analysis}

We performed the statistical analyses using Stata/IC 11 . Data for the patient characteristics are shown as the mean \pm SD. To investigate the difference between the eHbA1c and measured $\mathrm{HbA} 1 \mathrm{c}$ values, we calculated $95 \%$ confidence intervals $(\mathrm{CI})$ of the median of the difference using a bootstrap method (2000 bootstraps), and determined the $P$ values for the difference using the Wilcoxon signed-rank test.

\section{Results}

The clinical characteristics in each group are shown in Table 1. Patients in the HM group were less likely to have diabetes than patients in the other groups. The HbA1c, GA, hemoglobin, albumin, and eHbA1c levels were lower in the HM group than the other groups. The eGFR levels in the CRF and HD groups were lower than the other groups. Patients in the CRF and HD groups tended to have proteinuria and require erythropoietin or iron preparations.

In our previous study, we established the following equation: $\quad \mathrm{eHbA} 1 \mathrm{c}=0.216 \times \mathrm{GA}+2.978$ [3]. Figure 2 shows scatter plots for the HbA1c values versus the GA values for each group with a line for the equation.

In all the groups, the eHbAlc values (i.e., the line for the equation in Fig. 2) tended to be higher than the measured HbA1c levels. We further analyzed the medians of the differences between the eHbA1c and measured HbA1c values, and calculated the corresponding $95 \% \mathrm{CI}$ and $P$ values for each group (Table 2). In all the groups, the 


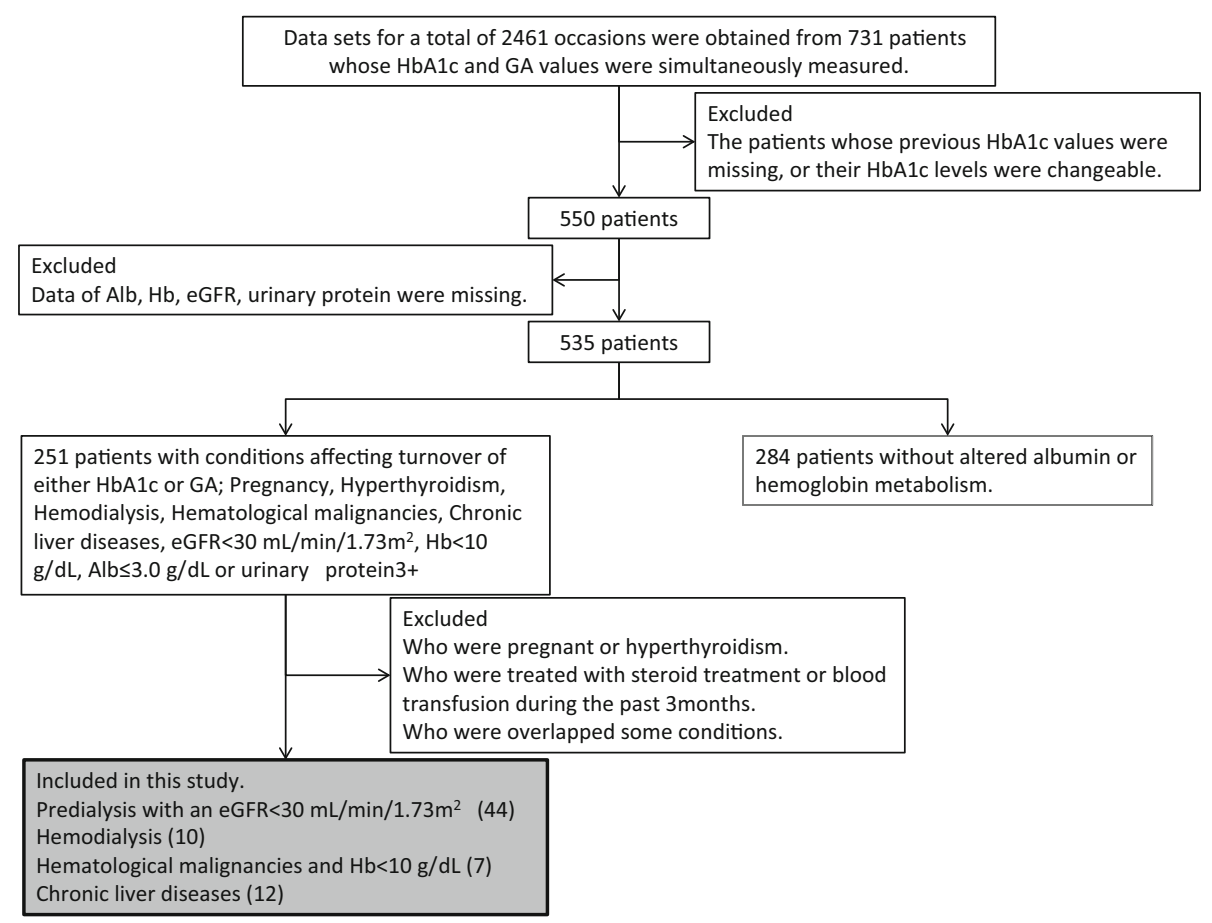

Fig. 1 Flow diagram depicting the study. Data sets for a total of 2461 occasions were obtained from 731 patients (including non-diabetes patients) whose $\mathrm{HbA1c}$ and GA values were simultaneously measured. If these values were measured in the patients on more than one occasion, the data set containing the smallest HbAlc value was selected. We then excluded patients whose previous $\mathrm{HbA1c}$ values were missing or whose $\mathrm{HbA} 1 \mathrm{c}$ levels were changeable, selecting 550 patients. We excluded patients without albumin, hemoglobin or eGFR data, and patients who had been treated with transfusions or steroids

eHbA1c values were significantly higher than the measured HbA1c levels. The median of the difference was $0.75 \%$ (95\% CI $0.40-1.10 \%, P$ for the difference is $<0.001$ ) in the CRF group, $0.80 \%(95 \%$ CI $0.30-1.65 \%, P$ for the difference is 0.041 ) in the HD group, $0.90 \%$ (95\% CI $0.90-1.30 \%, P$ for the difference is 0.028 ) in the HM group, $0.85 \%$ (95\% CI $0.40-1.50 \%, P$ for the difference is 0.009 ) in the CLD group.

\section{Discussion}

In this study, we calculated the eHbA1c value using an equation for each of the several groups of patients suffering from various diseases, and investigated the difference from the measured HbAlc values. Few studies have investigated the difference between estimated values and actual measurements of HbAlc.

The patients were classified into 4 groups as follows: 44 patients with chronic renal failure, 10 patients undergoing hemodialysis, 7 patients suffering from hematological malignancies and who had a hemoglobin level of less than within the previous 3 months. Finally, we included 44 predialysis patients with an eGFR of less than $30 \mathrm{~mL} / \mathrm{min} / 1.73 \mathrm{~m}^{2}$ (CRF), 10 patients who were undergoing hemodialysis (HD), 7 patients with hematological malignancies and their hemoglobin level of less than $10 \mathrm{~g} / \mathrm{dL}$ (HM), and 12 patients with chronic liver diseases (CLD). We further excluded patients who had combinations of these diseases, since the aim of this study was to investigate the impact of each condition affecting the turnover of either HbAlc or GA on the direction and magnitude of the discrepancy

$10 \mathrm{~g} / \mathrm{dL}$, and 12 patients who were suffering from chronic liver diseases. In all of the groups, the eHbA1c values were significantly higher than the measured $\mathrm{HbAlc}$ values. These results suggested that the measured $\mathrm{HbA1c}$ values in these groups may be underestimated in clinical practice.

In cases with chronic renal failure, renal anemia lowers the $\mathrm{HbAlc}$ values because the lifespan of the erythrocytes is shortened. The HbAlc and eGFR values are reportedly correlated with the lifespan of the erythrocytes in patients with diabetic nephropathy [8]. It has also been reported that the values of HbAlc are underestimated in patients with diabetic nephropathy undergoing peritoneal dialysis or hemodialysis [9]. Furthermore, the HbA1c values in patients who were treated with erythropoietin were lower than those patients who were not treated, since the life span of the erythrocytes is shortened [10]. Because renal anemia is unlikely to affect the GA value, GA may be useful in patients with renal anemia. Although $\mathrm{HbA1c}$ has been commonly measured, several professional societies (e.g., the Japanese Society for Dialysis Therapy [11] ) now recommend GA measurements for such patients. Our findings further suggest that eHbA1c may be a useful marker for the 
Table 1 Clinical characteristics in each groups

\begin{tabular}{|c|c|c|c|c|}
\hline & $\begin{array}{l}\text { Predialysis with an eGFR } \\
<30 \mathrm{~mL} / \mathrm{min} / 1.73 \mathrm{~m}^{2}(n=44)\end{array}$ & $\begin{array}{l}\text { Hemodialysis } \\
(n=10)\end{array}$ & $\begin{array}{l}\text { Hematological malignancies } \\
\text { and } \mathrm{Hb}<10 \mathrm{~g} / \mathrm{dL}(n=7)\end{array}$ & Chronic liver diseases $(n=12)$ \\
\hline $\operatorname{Men}(n)$ & 35 & 8 & 5 & 6 \\
\hline Age (years) & $66.8 \pm 12.0$ & $67.8 \pm 11.7$ & $69.3 \pm 18.2$ & $71.5 \pm 10.3$ \\
\hline HbA1c (\%) & $6.8 \pm 1.3$ & $6.4 \pm 0.9$ & $5.7 \pm 0.5$ & $7.1 \pm 0.8$ \\
\hline GA $(\%)$ & $20.8 \pm 5.7$ & $19.7 \pm 4.5$ & $16.1 \pm 2.0$ & $22.9 \pm 4.4$ \\
\hline $\mathrm{Hb}(\mathrm{g} / \mathrm{dL})$ & $11.3 \pm 1.8$ & $10.9 \pm 1.6$ & $8.7 \pm 0.8$ & $12.1 \pm 1.6$ \\
\hline Alb (g/dL) & $3.7 \pm 0.5$ & $3.6 \pm 0.9$ & $2.9 \pm 0.7$ & $3.7 \pm 0.4$ \\
\hline eHbA1c (\%) & $7.5 \pm 1.2$ & $7.3 \pm 1.0$ & $6.4 \pm 0.4$ & $7.9 \pm 1.0$ \\
\hline $\mathrm{eGFR}\left(\mathrm{mL} / \mathrm{min} / 1.73 \mathrm{~m}^{2}\right)$ & $16.6 \pm 7.8$ & - & $123.3 \pm 104.1$ & $69.0 \pm 14.4$ \\
\hline Diabetes $(n)$ & 43 & 9 & 2 & 11 \\
\hline Urinary protein $3+(n)$ & 13 & 4 & 0 & 0 \\
\hline Using erythropoietin $(n)$ & 19 & 6 & 0 & 0 \\
\hline Using iron preparation $(n)$ & 7 & 2 & 0 & $\begin{array}{l}1 \\
\text { Mean } \pm \text { SD }\end{array}$ \\
\hline
\end{tabular}

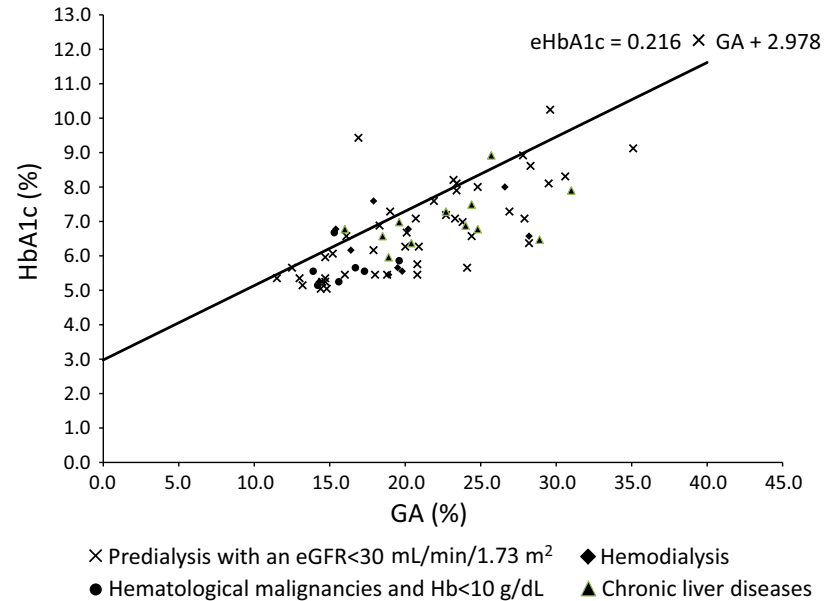

Fig. 2 Scatter plots for HbA1c values versus GA values in each group. In our previous study, we established the following equation: $\mathrm{eHbA} 1 \mathrm{c}=0.216 \times \mathrm{GA}+2.978$. Scatter plots for the HbA1c values versus the GA values are shown for each group with a line for the equation. In all the groups, the eHbA1c values tended to be higher values than the measured $\mathrm{HbAlc}$ levels

evaluation of glycemic control in patients with CRF or HD. However, a careful consideration is required in patients with diabetic nephropathy with marked proteinuria. The GA values are affected by the increased turnover of albumin metabolism and tend to decrease independent of glycemic state in patients with marked proteinuria [12], indicating their possible limited ability to evaluate glycemic control in such patients. Because the number of patients with marked proteinuria was relatively small in the present study, further studies are needed to clarify whether eHbAlc or GA is more useful than HbA1c in such patients.

In this study, we investigated 7 patients who were suffering from hematological malignancies and who had a
Table 2 The medians of the difference between eHbA1c and measured $\mathrm{HbA1c}$ values in each groups

\begin{tabular}{llll}
\hline & $\begin{array}{l}\text { The median of the } \\
\text { difference } \\
\text { between } \\
\text { eHbA1c and } \\
\text { measured HbA1c } \\
\text { values }(\%)\end{array}$ & & \\
& & & \\
& & & \\
& & $0.40-1.10$ & $<0.001$ \\
\hline $\begin{array}{l}\text { Predialysis with an eGFR } \\
<30 \mathrm{~mL} / \mathrm{min} / 1.73 \mathrm{~m}^{2} \\
(n=44)\end{array}$ & 0.75 & & \\
$\begin{array}{l}\text { Hemodialysis }(n=10) \\
\begin{array}{l}\text { Hematological } \\
\text { malignancies and } \mathrm{Hb} \\
<10 \mathrm{~g} / \mathrm{dL}(n=7)\end{array}\end{array}$ & 0.80 & $0.30-1.65$ & 0.041 \\
$\begin{array}{l}\text { Chronic liver diseases } \\
(n=12)\end{array}$ & 0.85 & $0.90-1.30$ & 0.028 \\
\hline
\end{tabular}

hemoglobin level of less than $10 \mathrm{~g} / \mathrm{dL}$. Both the measured $\mathrm{HbA1c}$ and the eHbA1c levels in the HM group were lower than those in the other groups. The lower frequency of patients with diabetes in the HM group may explain the lower $\mathrm{GA}$ and $\mathrm{eHbA1c}$ levels. HbA1c values are known to be low, relative to the glucose levels in patients with hemolytic anemia because the lifespan of the erythrocytes is shortened in patients with this condition [6]. Moreover, in patients with iron deficiency anemia, the HbA1c values tend to be higher than in healthy individuals but decrease after iron treatment [13]. Although the mechanisms remain to be investigated, the altered lifespan of erythrocytes may partially explain the difference between the measured $\mathrm{HbA} 1 \mathrm{c}$ and eHbA1c levels in the HM group observed in this study.

In chronic liver diseases, such as chronic hepatitis and liver cirrhosis, hypersplenism lowers the HbAlc values because of the shortened lifespan of the erythrocytes, 
whereas, it raises the GA values because of reduced albumin synthesis and the prolonged half-life of serum albumin $[5,14]$. Although neither marker reflects the plasma glucose control status accurately, we found the eHbA1c values were significantly higher than the measured $\mathrm{HbAlc}$ values in the CLD group.

Our study had several limitations. First, we retrospectively selected patients in whom simultaneous HbA1c and GA measurements had been obtained. Thus, a selection bias may exist. We excluded the patients, whose previous $\mathrm{HbA1c}$ values were missing or their $\mathrm{HbA} 1 \mathrm{c}$ levels were changeable, but we couldn't exclude the patients who had become good control over past few weeks. Second, as the data were collected from a single hospital and the GA values were not standardized, the present results might not be directly applicable to other hospitals. Third, the small sample size might limit the applicability of the findings. In clinical situation, patients with various conditions affect the GA values, so we should take consideration to use the equation of the eHbAlc.

In conclusion, we found that the measured $\mathrm{HbA1c}$ values were lower than the eHbA1c values in groups of patients with chronic renal failure, who were undergoing hemodialysis, suffering from hematological malignancies and had a hemoglobin level of less than $10 \mathrm{~g} / \mathrm{dL}$, and who had chronic liver diseases.

Acknowledgments This work was funded by health sciences research grants (Comprehensive Research on Life-style Related Diseases Including Cardiovascular Diseases and Diabetes Mellitus H22019 and H25-016) from the Ministry of Health, Labour and Welfare of Japan.

Conflict of interest None of the authors have any potential conflicts of interest associated with this research.

Open Access This article is distributed under the terms of the Creative Commons Attribution 4.0 International License (http:// creativecommons.org/licenses/by/4.0/), which permits unrestricted use, distribution, and reproduction in any medium, provided you give appropriate credit to the original author(s) and the source, provide a link to the Creative Commons license, and indicate if changes were made.

\section{References}

1. International Expert Committee. International Expert Committee report on the role of the A1C assay in the diagnosis of diabetes. Diabetes Care. 2009;32:1327-34.

2. Tahara $Y$, et al. Kinetics of HbA1c, glycated albumin, and fructosamine and analysis of their weight functions against preceding plasma glucose level. Diabetes Care. 1995;18:440-7.

3. Inoue $\mathrm{K}$, et al. Correlation between HbA1c and glycated albumin and a proposal for a novel conversion equation. Endocr $\mathrm{J}$. 2014;61(6):553-60.

4. Chujo K, et al. Indicators for blood glucose control in diabetics with end-stage chronic renal disease: $\mathrm{GHb}$ vs. glycated albumin (GA). J Med Invest. 2006;53:223-8.

5. Koga M, et al. CLD (chronic liver diseases)-HbA1C as a suitable indicator for estimation of mean plasma glucose in patients with chronic liver diseases. Diabetes Res Clin Pract. 2008;81:258-62.

6. Koga M, et al. Usefulness of glycated albumin as an indicator of glycemic control status in patients with hemolytic anemia. Clin Chim Acta. 2011;412:253-7.

7. Kashiwagi A, et al. International clinical harmonization of glycated hemoglobin in Japan: from Japan diabetes society to national glycohemoglobin standardization program values. Diabetol Int. 2012;3:8-10.

8. Shima $\mathrm{K}$, et al. Lower value of glycated haemoglobin relative to glycaemic control in diabetic patients with end-stage renal disease not on haemodialysis. Ann Clin Biochem. 2012;49:68-74.

9. Barry IF, et al. Comparison of glycated albumin and hemoglobin A1c concentrations in diabetic subjects on peritoneal and hemodialysis. Perit Dial Int. 2008;30:72-9.

10. Inaba M, et al. Glycated Albumin is a better glycemic indicator than glycated hemoglobin values in hemodialysis patients with diabetes: effect of anemia and erythropoietin injection. J Am Soc Nephrol. 2007;18:896-903.

11. The Japanese Society for Dialysis Therapy. Management of diabetic patients on hemodialysis 2012. Nihon Toseki Igakkai Zasshi. 2013;46(3):311-57.

12. Okada $\mathrm{T}$, et al. Influence of proteinuria on glycated albumin values in diabetic patients with chronic kidney disease. Intern Med. 2011;50:23-9.

13. Coban E, et al. Effect of iron deficiency anemia on the levels of hemoglobin A1c in nondiabetic patients. Acta Haematol. 2004; 112(3):126-8.

14. Bando $\mathrm{Y}$, et al. Association of serum glycated albumin to haemoglobin A1C ratio with hepatic function tests in patients with chronic liver disease. Ann Clin Biochem. 2009;46:368-72. 